\title{
The European Prevention of Alzheimer's Dementia (EPAD) Longitudinal Cohort Study: Baseline Data Release V500.0
}

\author{
C.W. Ritchie ${ }^{1}$, G. Muniz-Terrera ${ }^{1}$, M. Kivipelto ${ }^{2}$, A. Solomon' ${ }^{2}$ B. Tom ${ }^{3}$, J.L. Molinuevo ${ }^{4}$ On Behalf of the \\ EPAD Consortium
}

1. University of Edinburgh, United Kingdom; 2. Karolinska Institute, Sweden; 3. University of Cambridge, United Kingdom; 4. Barcelona Beta Research Centre, Spain

Corresponding Author: Craig William Ritchie, University of Edinburgh, United Kingdom, craig.ritchie@ed.ac.uk

J Prev Alz Dis 2020;1(7):8-13

Published online November 26, 2019, http:/ /dx.doi.org/10.14283/jpad.2019.46

\begin{abstract}
BACKGROUND: The European Prevention of Alzheimer's Dementia (EPAD) Programme is a pan-European project whose objective is to deliver a platform, adaptive, Phase 2 proof of concept $(\mathrm{PoC})$ trial for the secondary prevention of Alzheimer's dementia. A component of this platform is the Longitudinal Cohort Study (LCS) which acts as a readiness cohort for the PoC Trial as well as generating data for disease modelling work in the preclinical and prodromal phases of Alzheimer's dementia.

OBJECTIVES: The first data wave has been collected, quality checked, released and now available for analysis to answer numerous research questions. Here we describe the results from key variables in the EPAD LCS with the objective of using these results to compliment analyses of these data in the future.

DESIGN: EPAD LCS is a cohort study whose primary objective is as a readiness cohort for the EPAD PoC Trial. As such recruitment is not capped at any particular number but will continue to facilitate delivery of the EPAD PoC Trial. Research Participants are seen annually (with an additional 6 month visit in the first year).

SETTING: The EPAD Trial Delivery Network comprises
\end{abstract} currently 21 centres across Europe.

PARTICIPANTS: Research participants are included if they are over 50 years old and do not have a diagnosis of dementia.

Measurements: All research participants undergo multiple assessments to fully characterise the biology of Alzheimer's disease and relate this to risk factors (both fixed and modifiable) and biomarker expression of disease through brain imaging, fluid samples (CSF, blood, urine and saliva), cognitive performance, functional abilities and neuropsychiatric symptomatology.

RESULTS: V500.0 represents the first 500 research participants baselined into EPAD LCS. The mean age was $66.4(\mathrm{SD}=6.7)$ and $47.8 \%$ were male. The data was split for presentation into 4 groups: [1] $\mathrm{CDR}=0$ and Amyloid + (preclinical AD), [2] $C D R=0$ and Amyloid -, [3] CDR=0.5 and Amyloid + (prodromal AD) and [4] CDR=0.5 and Amyloid -.

CONCLUSIONS: The EPAD LCS is achieving its primary objective of trial readiness and the structured approach to data release as manifest by this first data release of V500.0 will assist researchers to describe and compare their findings as well as in systematic reviews and meta-analyses. It is anticipated given current recruitment rates that V1500.0 data release will take place in Autumn 2019. V500.1 (when the 1 year follow up is completed on the V500.0 (sub)cohort will be in Autumn 2019 also.
Key words: EPAD, cohort, Alzheimer's disease, prevention, disease modelling.

\section{Background}

The European Prevention of Alzheimer's Dementia (EPAD) project was initiated in L January 2015 and is funded by the Innovative Medicines Initiative. The overall project background and objectives are described elsewhere (1). In summary EPAD has a singular objective and that is to develop an entire infrastructure for the delivery of the EPAD Proof of Concept Trial. The EPAD PoC Trial is a platform trial, which employs a single master protocol with multiple appendices (representing each intervention) that uses Bayesian Adaptive Designs to develop interventions for the secondary prevention of Alzheimer's dementia through Phase 2. The EPAD Infrastructure has many components including a virtual register of people in partnering parent cohorts across Europe, the Trial Delivery Centre (TDC) network, the PoC trial platform of TDCs, vendors and Clinical Research Organisations and the Longitudinal Cohort Study (LCS). The primary objective of the EPAD LCS is as a readiness cohort for the PoC to minimise screen failure by way of detailed characterisation of research participants within it and to provide run-in data to be used to compare with postrandomisation data from the PoC itself. In accumulating vast amounts of data from a very large and highly characterised cohort, the EPAD LCS will be able to deliver data (eventually on an open data access platform) to the entire research community to assist with disease modelling and knowledge generation regarding the interplay between risk factors, brain disease, expression of brain disease (through biomarkers, cognition, function and neuropsychiatric symptoms) and how these change over time. Aware of the potential power of these data in the understanding of preclinical and prodromal Alzheimer's disease, the management of data releases to the research community had to be measured, transparent and organised. Moreover, all data and sample collection and sample analysis has been conducted to the highest 


\begin{tabular}{|c|c|c|c|c|c|}
\hline & $\begin{array}{l}\text { Total sample } \\
\quad(\mathrm{n}=500)\end{array}$ & $\begin{array}{c}\text { CDR0 } \\
\text { Amyloid- }(\mathrm{n}=282)\end{array}$ & $\begin{array}{c}\text { CDR0 } \\
\text { Amyloid+ }(n=119)\end{array}$ & $\begin{array}{c}\text { CDR0.5 } \\
\text { Amyloid- }(\mathrm{n}=49)\end{array}$ & $\begin{array}{c}\text { CDR0.5 } \\
\text { Amyloid }+(n=38)\end{array}$ \\
\hline Age (mean,SD) & $66.4(6.7)$ & $65.1(5.9)$ & $65.9(6.5)$ & $69.6(7.3)$ & $71.9(6.5)$ \\
\hline \multicolumn{6}{|l|}{ Gender } \\
\hline Female & $261(52.2 \%)$ & $154(54.6 \%)$ & $60(50.4 \%)$ & $25(51 \%)$ & $13(34.2 \%)$ \\
\hline Male & $239(47.8 \%)$ & $128(45.4 \%)$ & $59(49.6 \%)$ & $24(49 \%)$ & $25(65.8 \%)$ \\
\hline \multicolumn{6}{|l|}{ Marital Status } \\
\hline Married/cohabiting & $377(75.4 \%)$ & $221(78.4 \%)$ & $89(74.8 \%)$ & $34(69.4 \%)$ & $27(71 \%)$ \\
\hline Divorced & $55(11 \%)$ & $24(8.5 \%)$ & $15(12.7 \%)$ & $9(18.4 \%)$ & $6(15.8 \%)$ \\
\hline Single & $36(7.2 \%)$ & $19(6.7 \%)$ & $6(5 \%)$ & $4(8.2 \%)$ & $3(7.9 \%)$ \\
\hline Widowed & $32(6.4 \%)$ & $18(6.4 \%)$ & $9(7.6 \%)$ & $2(4.0 \%)$ & $2(5.3 \%)$ \\
\hline Years of Education (mean,SD) & $14.0(3.7)$ & $14.2(3.6)$ & 13.8(3.8) & 13.7(3.8) & 14.1(3.9) \\
\hline \multicolumn{6}{|l|}{ Family History } \\
\hline \multicolumn{6}{|l|}{$(\mathrm{n}, \%)$} \\
\hline Yes & $294(58.8 \%)$ & $182(64.5 \%)$ & $85(71.4 \%)$ & $8(16.3 \%)$ & $11(28.9 \%)$ \\
\hline No & $206(41.2 \%)$ & $100(35.5 \%)$ & $34(28.6 \%)$ & $41(83.7 \%)$ & $27(71.1 \%)$ \\
\hline \multicolumn{6}{|l|}{ ApoE4 Status (n,\%) } \\
\hline ApoEe4/- & $286(57.2 \%)$ & $99(35.1 \%)$ & $59(49.6 \%)$ & $10(20.4 \%)$ & $21(55.3 \%)$ \\
\hline ApoEe4/4 & $191(38.2 \%)$ & $172(61)$ & $59(49.6 \%)$ & $35(71.4 \%)$ & $16(42.1 \%)$ \\
\hline Unknown & $23(4.6)$ & $11(3.9 \%)$ & $1(0.8 \%)$ & $4(8.2 \%)$ & $1(2.6 \%)$ \\
\hline
\end{tabular}

\section{GLP and GCP standards.}

The V500.0 data release represents the first formal data release from the EPAD project for use by multiple researchers. This paper describes this data in detail to assist current and future researchers with their analysis and also to facilitate between project comparisons of data in systematic reviews and meta-analysis.

\section{Methods}

The EPAD LCS Protocol and Methodology is provided in detail elsewhere (2). The EPAD LCS has as its primary objective to be a readiness cohort for the EPAD PoC Trial. Therefore, recruitment into LCS is not capped and will continue ad infinitum to provide the necessary number of suitable research participants for the EPAD PoC Trial. The secondary objective of the EPAD LCS is to use the data generated for disease modelling. After consent, research participants complete a comprehensive series of assessments.

Research Participants are eligible for inclusion if they are over the age of 50 and do not have a diagnosis of dementia. They must also be deemed suitable in principal for later inclusion in a clinical trial and therefore should not have any medical or psychiatric disorders which would normally exclude people from such trials.

Research Participants are seen every year where the entire protocol of assessments is completed. There is also a 6-month visit where only cognition is assessed. The domains of assessment are [1] cognition, [2] neuroimaging, [3] fluid biomarkers, [4] genetics, [5] lifestyle, [6] clinical and psychiatric assessment, [7] neuropsychiatric symptoms, [8] function and [9] basic demography. The decisions on which outcome measures to use were subject to intense deliberation and review of the extant scientific literature by four EPAD Scientific Advisory Groups on Cognition and Clinical Outcomes, Biomarkers, Neuroimaging and Genetics.

Data releases from the EPAD LCS will be highly systematised. In our chosen nomenclature (V500.0): $\mathrm{V}=$ version, 500 is the number of sequentially recruited research participants and '. 0 ' refers to the data including only the baseline (visit 0 ) data. This subcohort will be followed over time so that the next release from this cohort V500.1 will take place in approximately 12 months. This will include all the baseline, 6 month and 12 month data from these 500 research participants. The '. 1 ' refers to the data including all data up until the 1 year visit, V500.2 will be when all data up until the 2 year visit is released.

\section{Cognition and Clinical Outcomes}

The Cognition and Clinical Scientific Advisory Group advised the LCS protocol authors on the construction of the EPAD Cognitive Examination (ECE) $(3,4)$ as well as on functional outcomes and the capturing of key neuropsychiatric features namely sleep, anxiety and depression. The cognitive outcomes captured are: RBANS $(5,6)$ (Primary Outcome Measure for EPAD 


\begin{tabular}{|c|c|c|c|c|c|}
\hline & $\begin{array}{l}\text { Total Sample } \\
(n=500)\end{array}$ & $\begin{array}{c}\text { CDR 0 } \\
\text { Amyloid - }(\mathrm{n}=282)\end{array}$ & $\begin{array}{c}\text { CDR 0 } \\
\text { Amyloid + (n=119) }\end{array}$ & $\begin{array}{c}\text { CDR } 0.5 \\
\text { Amyloid - }(n=49)\end{array}$ & $\begin{array}{c}\text { CDR } 0.5 \\
\text { Amyloid }+(n=38)\end{array}$ \\
\hline RBANS Total (mean/SD) & 103.1(12.7) & $104.7(12.0)$ & $104.4(10.5)$ & 100.1(14.7) & $91.7(14.5)$ \\
\hline RBANS DMI (mean/SD) & $102.5(13.5)$ & $103.7(11.5)$ & $104.0(12.3)$ & $102.9(14.9)$ & 90.1(19.3) \\
\hline RBANS List Learning & $28.2(4.7)$ & 28.6(4.4) & $29.3(4.2)$ & $26.9(4.6)$ & $23.0(5.4)$ \\
\hline RBANS Story Memory & $18.2(3.1)$ & $18.5(2.7)$ & $18.7(2.9)$ & $17.1(3.5)$ & $15.8(4.4)$ \\
\hline RBANS Figure Recall & $14.2(3.9)$ & $14.4(3.6)$ & $15.0(3.3)$ & $13.5(4.2)$ & $10.6(4.8)$ \\
\hline RBANS Figure Copy & 18.6(1.9) & 18.6(2.0) & 18.6(1.6) & $18.8(1.9)$ & $18.2(1.8)$ \\
\hline RBANS Line Orientation & $18.0(2.2)$ & $18.0(2.2)$ & $18.4(1.8)$ & $17.6(3.1)$ & $17.6(2.1)$ \\
\hline RBANS Picture Naming & $9.8(0.9)$ & $9.8(0.7)$ & $9.9(0.4)$ & $9.3(2.3)$ & $9.8(0.6)$ \\
\hline RBANS Semantic Fluency & $19.2(5.6)$ & $19.8(5.4)$ & $19.4(5.5)$ & $17.1(6.7)$ & $16.6(5.0)$ \\
\hline RBANS Digit Span & 9.5(2.3) & $9.6(2.3)$ & $9.6(2.3)$ & $9.2(1.9)$ & $8.6(2.1)$ \\
\hline RBANS Coding & $43.9(10.8)$ & $46.6(9.7)$ & $43.6(9.1)$ & 37.1(13.5) & $34.0(10.6)$ \\
\hline MMSE (Mean/SD) & 28.6(1.6) & 28.8(1.5) & $28.8(1.3)$ & 28.3(1.9) & $27.6(1.7)$ \\
\hline
\end{tabular}

PoC Trial), CDR (7), MMSE (8), NIH Toolbox tests (Dot Counting, Flanker) (9, 10), UCSF Brain Health Assessment (Favorites) (11), Supermarket Trolley Test (12) and Four Mountains Test (13). Function is assessed using the Amsterdam Instrumental Activity of Daily Living Assessment $(14,15)$. Sleep is assessed using the Pittsburgh Sleep Questionnaire (16); Anxiety is measured using the State/Trait Anxiety Inventory (17) and Depression using the Geriatric Depression Scale $(18,19)$.

All cognitive and clinical data is captured on tablets (either on the Medavante Virgil Platform or the UCSF Tabcat System). These data are then uploaded to the EPAD LCS Master Database held by the EPAD LCS Clinical Research Organisation IQVIA for conciliation with other data sources e.g. imaging and eCRF data before being quality controlled and then pushed to the Analytical Database hosted by the EPAD Partner Aridhia.

\section{Neuroimaging Outcomes}

The Neuroimaging Scientific Advisory Group advised the LCS protocol authors on structural and functional MRI based evaluations optimised for understanding brain changes in preclinical and prodromal Alzheimer's disease (20). The structural sequence captured in the protocol were Cortical thickness, deep grey matter volumes, fractional anisotropy of temporal lobe, diffusion kurtosis (multi b-valueDTI) and network alterations. The functional MRI outcomes were global \& parietal CBF and changes within the default-mode network \& relation with hippocampal activity(rsfMRI), Bolus arrival time (multi-delay arterial spin labelling) and network analysis (rsfMRI) though not all of these data have been analysed as yet in V500.0 and therefore not presented in this paper.

All brain-imaging facilities are accredited by the EPAD LCS Imaging CRO IXICO. Imaging files from the site are transferred to IXICO for central reading and safety evaluation. Key outcomes are then transferred to the Master Database for conciliation with other data feeds before these data are transferred to Aridhia and the EPAD Analytical Database. MRI scanners are a minimum of $1.5 \mathrm{~T}$.

\section{Biomarker Outcomes}

The Biomarker Scientific Advisory Group after review of the existing evidence around neuropathological changes in preclinical and prodromal AD had to decide which biomarkers were either fully validated as markers of disease or remained at the discovery phase of development. The former were to be incorporated in the protocol whilst the potential to explore the others would be reserved to a future date from EPAD LCS samples collected, shipped and stored under optimal conditions. The Biomarker SAG therefore also oversaw the creation of the laboratory manual (available on line at www.ep-ad. org).

The only biomarkers in the protocol are CSF ABeta, Tau and Phosphorylated Tau. All samples are shipped from sites and stored centrally at the EPAD BioBank at the University of Edinburgh before CSF samples taken in Sarstedt tubes are shipped to the University of Gothenburg, Sweden for analysis using the Roche Diagnostics Elecsys Platform. Results are then forwarded to the IQVIA Master Database and then transferred to the Aridhia Analytical Database. Using this system a threshold of 1,000 pg $/ \mathrm{ml}$ of ABeta42 was agreed upon to define amyloid positivity.

Saliva (drooling sample and salivette), urine and plasma are also stored in the EPAD BioBank for future use. To date none of these samples have been analysed.

\section{Genetics Outcomes}

The Genetics Scientific Advisory Group had a similar remit to that of the Biomarker SAG in so much as they agreed on recommendations for outcomes to be done in all samples and within the protocol and advise on 


\begin{tabular}{|c|c|c|c|c|c|}
\hline & $\begin{array}{l}\text { Total Sample } \\
\quad(\mathrm{n}=500)\end{array}$ & $\begin{array}{c}\text { CDR 0 } \\
\text { Amyloid - }(\mathrm{n}=282)\end{array}$ & $\begin{array}{c}\text { CDR } 0 \\
\text { Amyloid + (n=119) }\end{array}$ & $\begin{array}{c}\text { CDR } 0.5 \\
\text { Amyloid }-(n=49)\end{array}$ & $\begin{array}{c}\text { CDR } 0.5 \\
\text { Amyloid }+(n=38)\end{array}$ \\
\hline \multicolumn{6}{|l|}{ Schelten's Score [Right] } \\
\hline 0 & $338(67.6 \%)$ & $209(74.1 \%)$ & $83(69.8 \%)$ & $27(55.1 \%)$ & $17(44.7 \%)$ \\
\hline 1 & $119(23.8 \%)$ & $61(21.7 \%)$ & $31(26 \%)$ & $9(18.4 \%)$ & $17(44.7 \%)$ \\
\hline 2 & $21(4.2 \%)$ & $8(2.8 \%)$ & $4(3.4 \%)$ & $6(12.2 \%)$ & $2(5.4 \%)$ \\
\hline 3 & $7(1.4 \%)$ & 0 & $1(0.8 \%)$ & $5(10.2 \%)$ & $1(2.6 \%)$ \\
\hline 4 & 0 & 0 & 0 & 0 & 0 \\
\hline Missing & $15(3.0 \%)$ & $4(1.4 \%)$ & 0 & $2(4.1 \%)$ & $1(2.6 \%)$ \\
\hline \multicolumn{6}{|l|}{ Scheltens' Score [Left] } \\
\hline 0 & $347(69.4 \%)$ & $223(79.1 \%)$ & $78(65.5 \%)$ & $30(61.2 \%)$ & $14(36.8 \%)$ \\
\hline 1 & $113(22.6 \%)$ & $48(17.0 \%)$ & $39(32.8 \%)$ & $8(16.3 \%)$ & $17(44.8 \%)$ \\
\hline 2 & $19(3.8 \%)$ & $7(2.5 \%)$ & $2(1.7 \%)$ & $5(10.2 \%)$ & $4(10.5 \%)$ \\
\hline 3 & $5(1 \%)$ & 0 & 0 & $3(6.2 \%)$ & $2(5.3 \%)$ \\
\hline 4 & $1(0.2 \%)$ & 0 & 0 & $1(2.0 \%)$ & 0 \\
\hline Missing & $15(3 \%)$ & $4(1.4 \%)$ & 0 & $2(4.1 \%)$ & $1(2.6 \%)$ \\
\hline \multicolumn{6}{|l|}{ Fazekas' Score (Deep White Matter) } \\
\hline 0 & $129(25.8 \%)$ & $83(29.4 \%)$ & $29(24.4 \%)$ & $10(20.4 \%)$ & $6(15.8 \%)$ \\
\hline 1 & $267(53.4 \%)$ & $149(52.9 \%)$ & $65(54.6 \%)$ & $32(65.3 \%)$ & $19(50 \%)$ \\
\hline 2 & $78(15.6 \%)$ & $44(15.6 \%)$ & $17(14.3 \%)$ & $5(10.2 \%)$ & $11(29 \%)$ \\
\hline 3 & $11(2.2 \%)$ & $2(0.7 \%)$ & $8(6.7 \%)$ & 0 & $1(2.6 \%)$ \\
\hline Missing & $15(3 \%)$ & $4(1.4 \%)$ & 0 & $2(4.0 \%)$ & 1(2.6) \\
\hline \multicolumn{6}{|l|}{ Fazekas' Score (Periventricular White Matter) } \\
\hline 0 & $273(54.6 \%)$ & $168(59.6 \%)$ & $62(52.1 \%)$ & $28(57.1 \%)$ & $12(31.6 \%)$ \\
\hline 1 & $136(27.2 \%)$ & $72(25.5 \%)$ & $40(33.6 \%)$ & $13(26.6 \%)$ & $11(29 \%)$ \\
\hline 2 & $67(13.4 \%)$ & $35(12.4 \%)$ & $14(11.8 \%)$ & $5(10.2 \%)$ & $12(31.6 \%)$ \\
\hline 3 & $9(1.8 \%)$ & $3(1.1 \%)$ & $3(2.5 \%)$ & $1(2.0 \%)$ & $2(5.2 \%)$ \\
\hline Missing & $15(3 \%)$ & $4(1.4 \%)$ & 0 & $2(4.1)$ & $1(2.6 \%)$ \\
\hline Hippocampal Volume $\mathrm{R} \mathrm{mm}^{3}$ (Mean/SD) & $2430.0(328.5)(\mathrm{n}=480)$ & $2449.2(293.0)(n=274)$ & $2493.0(303.6)(\mathrm{n}=118)$ & $2370(308.2)(n=47)$ & $2208.9(496.9)(\mathrm{n}=37)$ \\
\hline \multirow[t]{2}{*}{ Hippocampal Volume L mm³ (Mean/SD) } & 2354.6(318.0) & $2385.0(282.4)$ & 2395.0(295.5) & $2267.5(295.0)$ & 2139.2(493.4) \\
\hline & Total Sample $(\mathrm{n}=500)$ & $\begin{array}{c}\text { CDR 0 } \\
\text { Amyloid - }(\mathrm{n}=282)\end{array}$ & $\begin{array}{c}\text { CDR 0 } \\
\text { Amyloid + (n=119) }\end{array}$ & $\begin{array}{c}\text { CDR } 0.5 \\
\text { Amyloid }-(n=49)\end{array}$ & $\begin{array}{c}\text { CDR } 0.5 \\
\text { Amyloid }+(n=37)\end{array}$ \\
\hline Whole Brain Volume $\mathrm{mm}^{3}$ (Mean/SD) & $1103866(108312.2)$ & $1102517(107156.7)$ & 1118615 (108874.4) & 1082654 (111207.5) & $1093971(107907.2)$ \\
\hline Ventricular Volume $\mathrm{mm}^{3}$ (Mean/SD) & $27706.6(16787.3)$ & $24071.6(13808.3)$ & $30933.2(18407.2)$ & $29798.0(16570.9)$ & $40984.38(22468.8)$ \\
\hline $\begin{array}{l}\text { Pseudo total intracranial volume factor } \\
\text { (Mean/SD) }\end{array}$ & $0.8386016(0.0904448)$ & $0.830509(0.0904519)$ & $0.8429424(0.0906328)$ & $0.8398999(0.0867448)$ & $0.8762338(0.0892778)$ \\
\hline $\begin{array}{l}\text { White Matter Lesion Volume } \mathrm{mm}^{3} \\
\text { (Mean/SD) }\end{array}$ & $17979.3(41513.7)$ & $12283.9(33927.4)$ & $32729.0(54488.1)$ & $8920.9(20631.2)$ & $25656.7(53550.6)$ \\
\hline
\end{tabular}

optimal storage for future use. They recommended that all samples should be tested for ApoE status of the research participants. Sampling preparation and storage details can be found in the EPAD lab manual (available on line at www.ep-ad.org)

Taqman Genotyping was carried out in a single laboratory on QuantStudio12K Flex to establish ApoE variants. Genomic DNA was isolated from whole blood and genotyping was performed in 384 well-plates, using the TaqMan polymerase chain reaction-based method. The final volume PCR reaction was $5 \mu \mathrm{l}$ using $20 \mathrm{ng}$ of genomic DNA, $2.5 \mu \mathrm{l}$ of Taqman Master Mix and $0.125 \mu$ l of 40x Assay By design Genotyping Assay Mix, or $0.25 \mu 1$ of $20 x$ Assay On Demand Genotyping Assay. The cycling parameters were $95^{\circ}$ for 10 minutes, followed by forty cycles of denaturation at $92^{\circ}$ for 15 seconds and annealing/extension at $60^{\circ}$ for 1 minute. PCR plates were then read on ThermoFisher QuantStudio 12K Flex Real Time PCR System instrument with QuantStudio 12K Flex Software or Taqman Genotyper Software v1.3. 


\begin{tabular}{|c|c|c|c|c|c|}
\hline & $\begin{array}{l}\text { Total Sample } \\
\quad(\mathrm{n}=447)\end{array}$ & $\begin{array}{c}\text { CDR 0 } \\
\text { Amyloid }-(n=252)\end{array}$ & $\begin{array}{c}\text { CDR 0 } \\
\text { Amyloid + (n=119) }\end{array}$ & $\begin{array}{c}\text { CDR } 0.5 \\
\text { Amyloid }-(n=37)\end{array}$ & $\begin{array}{c}\text { CDR } 0.5 \\
\text { Amyloid }+(n=38)\end{array}$ \\
\hline CSF ABeta & $1330.5(614.1)$ & $1660.6(490.2)$ & $726.1(194.2)$ & $1701.8(532.0)$ & $678.3(162.6)$ \\
\hline CSF pTau & $19.2(9.7)$ & $18.2(8.0)$ & 19.3(11.5) & $18.8(6.3)$ & $25.8(14.0)$ \\
\hline CSF TTau & 221.7(93.4) & $216.8(82.8)$ & 212.3(104.3) & $228.0(71.3)$ & 277.6(123.6) \\
\hline CSF pTau / ABeta Ratio & $0.018(0.017)$ & $0.0114(0.005)$ & $0.0293(0.0197)$ & $0.0117(0.0046)$ & $0.0422(0.0313)$ \\
\hline CSF TTau / ABeta Ratio & $0.2118(0.1699)$ & $0.1358(0.057)$ & $0.3187(0.1816)$ & $0.1417(0.0525)$ & $0.4498(0.2806)$ \\
\hline
\end{tabular}

\begin{tabular}{|c|c|c|c|c|c|}
\hline & $\begin{array}{l}\text { Total Sample } \\
\quad(\mathrm{n}=500)\end{array}$ & $\begin{array}{c}\text { CDR 0 } \\
\text { Amyloid - }(n=282)\end{array}$ & $\begin{array}{c}\text { CDR 0 } \\
\text { Amyloid + }(\mathrm{n}=119)\end{array}$ & $\begin{array}{c}\text { CDR } 0.5 \\
\text { Amyloid }-(n=49)\end{array}$ & $\begin{array}{c}\text { CDR } 0.5 \\
\text { Amyloid }+(n=38)\end{array}$ \\
\hline State Trait Anxiety Inventory & $64.5(15.7)(\mathrm{n}=483)$ & $62.4(14.5)(\mathrm{n}=276)$ & $64.8(16.3)(\mathrm{n}=119)$ & $68.4(14.9)(\mathrm{n}=48)$ & $73.0(16.0)(n=37)$ \\
\hline Pittsburgh Sleep Quality Index & $5.17(3.3)(n=485)$ & $4.96(3.3)(n=278)$ & $5.0(2.9)(\mathrm{n}=117)$ & $6.31(3.88)(\mathrm{n}=48)$ & $5.9(3.4)(n=38)$ \\
\hline Amsterdam IADL & $0.77(2.42)(\mathrm{n}=480)$ & $0.29(0.8)(n=273)$ & $0.36(0.89)(\mathrm{n}=118)$ & $1.71(3.7)(\mathrm{n}=45)$ & $4.48(5.93)(\mathrm{n}=38)$ \\
\hline
\end{tabular}

\section{Demographic, lifestyle, clinical and other outcomes}

The EPAD LCS Protocol authors decided upon all outcomes following advice from the four listed Scientific Advisory Groups. Other outcomes were decided solely by the protocol authors. Lifestyle factors captured were self reported physical activity, diet, smoking/alcohol/ drug behaviour. Demography included gender, age, years of education and race (where allowable by regional authorities to be captured). Clinical history and physical examination was captured in standardised source documents to cover all medical and psychiatric domains including medication use. Specific assessment of head injury was undertaken using the Brain Injuries Screening Questionnaire.

\section{Results}

All results are presented similarly as the total sample and by amyloid and CDR status. Some data on variables presented are missing and then within that grouping amyloid status may be missing too due to analysis problems.

Lifestyle variables and medical history of research participants are not presented here but available on line on the EPAD website.

\section{Discussion}

The objective of this paper was to convey the structure of the EPAD LCS using the first data release from the project. With a large cohort that has open ended and perpetual recruitment, it was considered crucial that the academic and broader research community could have clarity on which data sets are being used from EPAD that form the basis of secondary data analysis. It is not in the scope of this paper to draw any conclusions from the data as no research question is being proposed here or hypothesis being tested. However, in terms of readiness for the EPAD PoC trial, just under 35\% of the cohort are amyloid positive with the majority of these being $\mathrm{CDR}=0$ which probably reflects the initial source of recruitment to EPAD LCS from population based parent cohorts (21). To date $26.6 \%$ of the sample have preclinical Alzheimer's disease and $8.3 \%$ prodromal Alzheimer's disease. However with an increasing drive in recent months to significantly increase the proportion of people in the cohort with $\mathrm{CDR}=0.5$ (in $\mathrm{V} 500.0=14.8 \%$ ) to nearer $30 \%$, we expect confidently that by 2020 and commencement of the PoC trial, the LCS will have the necessary level of readiness. V500.0 also has a broad range of research participants in terms of many key outcomes that will assist in disease modelling work and other hypothesis testing.

The quality of the data, sample preparation, storage and analysis was of paramount concern to the EPAD Consortium and much effort has gone into this with lab and imaging manuals available on the EPAD website to give researchers both assurance and clarity on the processes undertaken in EPAD to deliver high quality data.

The value of the EPAD LCS will clearly increase as the sample size increases and greater length of follow up is achieved in each sub-cohort. Description of each data release from EPAD is planned and will follow this initial formatting.

\section{Conclusions}

The EPAD Programme is a very large and ambitious programme that is ultimately set to deliver a platform, multi-arm PoC trial to test interventions targeting the secondary prevention of Alzheimer's dementia. Whilst 
the project has numerous key components, the EPAD LCS is central to all the efforts. Data from this cohort will be of great value to the research community so managed, orderly, structured and well described data releases are critical as a reflection of the importance of this database and the time and effort all our research participants have committed and made to help understand Alzheimer's disease before dementia develops and in doing so move a step closer to its prevention.

\section{Conflict of interest: No conflict of interest.}

Funding: The European Prevention of Alzheimer's Dementia project has received support from the EU/EFPIA Innovative Medicines Initiative Joint Undertaking EPAD grant agreement number 115736.

Ethical Standards: The EPAD LCS protocol and materials are submitted to the Independent Ethics Committee or other relevant ethical review board for written approval as required by local laws and regulations. A copy of approval is required by the University of Edinburgh as Sponsor before the study commences at each site. The study is designed and conducted in accordance with the guidelines for Good Clinical Practice (GCP), and with the ethical principles as proclaimed in the Declaration of Helsinki. All participants are required to provide written informed consent prior to participation in any research activities laid out in the EPAD LCS protocol.

Open Access: This article is distributed under the terms of the Creative Commons Attribution 4.0 International License (http://creativecommons.org/ licenses/by/4.0/), which permits use, duplication, adaptation, distribution and reproduction in any medium or format, as long as you give appropriate credit to the original author(s) and the source, provide a link to the Creative Commons license and indicate if changes were made.

\section{References}

1. Ritchie CW, Molinuevo JL, Satlin A, Van Der Geyten, Lovestone S and Truyen L. The European Prevention of Alzheimer's Dementia (EPAD) Consortium: A platform to enable the secondary prevention of Alzheimer's Dementia through improved Proof of Concept Trials. Lancet Psychiatry 2016;3(2):179-86

2. Solomon A et al., The European Prevention of Alzheimer's Dementia Longitudinal Cohort Study (EPAD LCS): study protocol. BMJ Open. 2018;8:e021017

3. Mortamais M, Ash JA, Harrison J, Kaye J, Kramer J, Randolph C, Pose C, Albala B, Ropacki M, Ritchie CW, Ritchie K. Detecting cognitive changes in preclinical Alzheimer's disease: A review of its feasibility. Alzheimers Dement. 2017;13(4):468-92.

4. Ritchie K, Ropacki M, Albala B, Harrison J, Kaye J, Kramer J, Randolph C and Ritchie CW. Recommended cognitive outcomes in preclinical Alzheimer's disease: Consensus statement from the European Prevention of Alzheimer's Dementia project. Alzheimers Dement. 2017;13(2):186-195

5. Karantzoulis S, Novitski J, Gold M, Randolph C. The Repeatable Battery for the Assessment of Neuropsychological Status (RBANS): Utility in detection and characterization of mild cognitive impairment due to Alzheimer's disease. Arch Clin Neuropsychol. 2013;28(8):837-44.
6. England HB, Gillis MM, Hampstead BM. RBANS memory indices are related to medial temporal lobe volumetrics in healthy older adults and those with mild cognitive impairment. Arch Clin Neuropsychol. 2014;29(4):322-8.

7. Morris JC. The Clinical Dementia Rating (CDR): current version and scoring rules. Neurol. 1993;43:2412-2414

8. Folstein MF, Folstein SE, McHugh PR. "Mini-mental state": a practical method for grading the cognitive state of patients for the clinician. J Psychiatr Res. 1975;12(3):189-98

9. Kramer JH, Mungas D, Possin KL, Rankin KP, Boxer AL, Rosen HJ, Bostrom A, Sinha L, Berhel A, Widmeyer M. NIH EXAMINER: conceptualization and development of an executive function battery. J Int Neuropsychol Soc. 2014;20(1):11-9.

10. Weintraub S, Dikmen SS, Heaton RK, Tulsky DS, Zelazo PD, Bauer PI, Carlozzi NE, Slotkin J, Blitz D, Wallner-Allen K, Fox NA. Cognition assessment using the NIH Toolbox. Neurol. 2013;80(11 Supplement 3):S54-64.

11. Possin KL, Moskowitz T, Erlhoff SJ, Rogers KM, Johnson ET, Steele NZ, Higgins JJ, Stiver J, Alioto AG, Farias ST, Miller BL. The Brain Health Assessment for Detecting and Diagnosing Neurocognitive Disorders. J Am Geriatr Soc. 2018;66(1):150-6.

12. Tu S, Wong S, Hodges JR, Irish M, Piguet $\mathrm{O}$, Hornberger M. Lost in spatial translation-A novel tool to objectively assess spatial disorientation in Alzheimer's disease and frontotemporal dementia. Cortex. 2015;67:83-94.

13. Moodley K, Minati L, Contarino V, Prioni S, Wood R, Cooper R, D'incerti L, Tagliavini F, Chan D. Diagnostic differentiation of mild cognitive impairment due to Alzheimer's disease using a hippocampus-dependent test of spatial memory. Hippocampus. 2015;25(8):939-51

14. Sikkes SA, Knol DL, Pijnenburg YA, de Lange-de Klerk ES, Uitdehaag BM, Scheltens P. Validation of the Amsterdam IADL Questionnaire ${ }^{\circ}$, a new tool to measure instrumental activities of daily living in dementia. Neuroepidemiology. 2013;41(1): 35-41

15. Sikkes SA, de Lange-de Klerk ES, Pijnenburg YA, Gillissen F, Romkes R, Knol DL, Uitdehaag BM, Scheltens P. A new informant-based questionnaire for instrumental activities of daily living in dementia. Alzheimers Dement. 2012;8(6):536-43.

16. Buysse DJ, Reynolds III CF, Monk TH, Berman SR, Kupfer DJ. The Pittsburgh Sleep Quality Index: a new instrument for psychiatric practice and research. Psychiatry Res. 1989;28(2):193-213.

17. Julian LJ. Measures of anxiety: State-Trait Anxiety Inventory (STAI), Beck Anxiety Inventory (BAI), and Hospital Anxiety and Depression Scale-Anxiety (HADS-A). Arthritis Care Res. 2011;63(S11):S467-72.

18. Yesavage JA, Brink TL, Rose TL, Lum O, Huang V, Adey M, Leirer VO. Development and validation of a geriatric depression screening scale: a preliminary report. J Psychiatr Res. 1982;17(1):37-49.

19. Debruyne H, Van Buggenhout M, Le Bastard N, Aries M, Audenaert K, De Deyn PP, Engelborghs S. Is the geriatric depression scale a reliable screening tool for depressive symptoms in elderly patients with cognitive impairment? Int J Geriatr Psychiatry. 2009;24(6):556-62.

20. Ingala S, ten Kate M, Schwarz A, Fox N, Chetelat G, van Berckel B, Ewers M, Foley C, Gispert JD, Hill D, Irizarry M, Lammertsma A, Molinuevo JL, Ritchie CW, Scheltens P, Schmidt M, Visser PJ, Waldman A, Wardlaw J, Haller S, Barkhof F. Secondary prevention of Alzheimer's dementia - neuroimaging contributions. J Prev Alzheimers Dis. 2018;10(1):112

21. Vermunt L, Veal CD, ter Meulen L, Chrysostomou C, van der Flier W, Frisoni GB, Guessous I, Kivipelto M, Marizzoni M, Martinez-Lage P, Molinuevo JL, Porteus D, Ritchie K, Scheltens P, Ousset PJ, Ritchie CW, Luscan G, Brookes AJ, Visser PJ. European Prevention of Alzheimer's Dementia (EPAD) Registry: recruitment and pre-screening approach for a longitudinal cohort and prevention trials. Alzheimers Dement. 2018;14(6): 837-842 